\title{
Rehabilitation under Social Isolation: Outcomes of Older Individuals with Hip Fractures, Admitted to a Postacute Geriatric Rehabilitation Center, during the COVID-19 Pandemic
}

\author{
Yaara Leibovici-Weissman ${ }^{a, b}$ Yochai Levy ${ }^{a, b}$ Michal Michaelis ${ }^{a, b}$ \\ Avital Hershkovitz ${ }^{b, c}$ \\ ${ }^{a}$ Acute Geriatric Department, Beilinson Hospital, Rabin Medical Center, Petach Tikva, Israel; bSackler School of \\ Medicine, Tel Aviv University, Tel Aviv, Israel; 'Beit Rivka Geriatric Rehabilitation Center, Petach Tikva, Israel
}

\section{Keywords}

COVID-19 · Hip fractures · Rehabilitation · Social distance ·

Treatment outcome

\begin{abstract}
Background/Aims: The novel coronavirus SARS-CoV- 2 is the cause of an ongoing pandemic. The highest mortality rate is observed among the older adult population. During the first wave of the pandemic (March-June 2020), following a national health decree demanding that no visitors or family members be allowed in health institutions, our geriatric rehabilitation center closed gates to all visitors from the outside. We aimed to assess the rehabilitation outcomes of older patients with hip fractures in the first pandemic wave, who underwent rehabilitation under complete social isolation from primary care givers and family members. Methods: This was a retrospective cohort study. It took place at a universityaffiliated, major postacute geriatric rehabilitation center. Rehabilitation outcomes measured were discharge functional independence measure (FIM) score and motor FIM score, FIM score change, motor FIM score change, favorable motor FIM effectiveness, length of stay, discharge destination, and home aid at discharge. Results: The study group included 36
\end{abstract}

patients who were admitted during the first wave of the COVID-19 pandemic (March 1, 2020, to June 30, 2020). The control group comprises 106 individuals with hip fractures who were admitted in the respective time periods during the previous 2 years, that is, March 1, 2018, to June 30, 2018, and March 1, 2019, to June 30, 2019. The facility's occupancy rates were much lower during the first wave of the pandemic in comparison with previous years (78 and $99 \%$, respectively). Patients showed similar demographics and comorbidities, but the social isolation group was more severely deconditioned upon admission. All rehabilitation outcomes were similar between groups. Conclusion: According to the findings of this retrospective study, social isolation from family and caregivers did not have a detrimental effect on the rehabilitation outcomes of these patients. More evidence must be gathered and presented on the matter.

(c) 2021 S. Karger AG, Basel

\section{Introduction}

The novel coronavirus SARS-CoV-2 is the cause of an ongoing pandemic. Clinical presentation varies from asymptomatic carriage to severe disease, causing pneumo-

$\begin{aligned} & \text { karger@karger.com } \\ & \text { www.karger.com/ger }\end{aligned}$
Karger ${ }^{\prime /}$

Yaara Leibovici-Weissman

Acute Geriatric Department, Rabin Medical Center Beilinson Hospital, 39 Jabotinsky St.

Petach Tikva 4941492 (Israel)

yaaralk@gmail.com 
nia, acute respiratory distress syndrome, and septic shock $[1,2]$. The highest mortality rate is observed among the 65 years and older population $[3,4]$. During the first wave of the pandemic in Israel (from beginning of March till the end of June 2020), the Israeli government issued a national health decree [5], regulating important issues such as mandatory isolation and obligatory mask wearing in public. Regarding health institutions, the regulations demand that "no visitors, nor family members be allowed, unless under special authorization from management, while using personal protection equipment (PPE)." Our institute is a postacute geriatric rehabilitation center. With the first wave of the pandemic, in accordance with the national health decree issued by the government, our institute went into complete protective lockdown, not allowing any outside visitors to enter the facility. Hospitalized patients were not allowed any temporary leaves until final discharge. The goal was to protect the older, highrisk patients, from SARS-CoV-2. Hence, our patient population found itself in rehabilitation under social isolation from family and main caregivers. Families maintained contact with their loved ones primarily via telephone; face to face meetings were rarely conducted, with family members staying outside the facility, engaging with their relatives across the facility's outer fence, maintaining sufficient distance and using personal protection equipment. For 3 months, both the rehabilitation multidisciplinary staff and the patients themselves were operating in a new and uncharted territory.

There are voices in the medical community that raise concerns whether social isolation might be detrimental to the mental and physical health of the older population [6, 7], and evidence exists regarding the negative mental impact of the pandemic [8]. The presence of family members at the patient's bedside affects patient outcomes. Open visitations have been proven to hasten recovery times, decrease inhospital length of stay, and improve morale and satisfaction reported by the patients and their families [9]. Integrating families in the rehabilitation of acutely ill patients has been proven beneficial too. It improves the patient's rehabilitation outcomes, promotes family integrity, and even eases some of the burden of care experienced by the caring staff [10-12]. Many publications regarding rehabilitation during the COVID-19 pandemic are already available. Authors are calling rehabilitation services to prepare for the rising numbers of older convalescing patients, in need of early rehabilitation by a multidisciplinary team [13]. Other publications are drawing attention to the increasing need for inpatient rehabilitation, with emphasis on respiratory rehabilitation and optimizing functional status [14]. The unique circumstances created by the COVID-19 pandemic call for the rethinking of existing models, either by creating postacute rehabilitation facilities for patients who might still be contagious, or by creating supportive networks for home rehabilitation [15]. However, we did not find data regarding rehabilitation outcomes of patients under social isolation. With the next wave of SARS-CoV-2 already impacting many parts of the world, knowing how social isolation of older individuals with hip fractures in geriatric rehabilitation facilities influences their outcomes might offer value for policy makers and geriatric rehabilitation staff.

We aimed to compare outcomes of patients with hip fractures, admitted during the first wave of the COVID-19 pandemic, who experienced social isolation from their families and caregivers, to those of patients admitted on parallel periods during 2018 and 2019. We hypothesized that with a lack of support from family and main caregivers, the rehabilitation outcomes of patients with hip fractures will be inferior to those of patients admitted in previous years.

\section{Materials and Methods}

\section{Participants}

This retrospective cohort study was performed in a universityaffiliated, major postacute geriatric rehabilitation center. The center admits older patients from nearby major acute hospitals. Before the COVID-19 pandemic, during admission interviews, we encouraged patients' families to visit their relatives as frequently as possible and assist in their care during rehabilitation. Many patients had a family member assisting during physical and occupational therapy sessions. Patients who already had a main caregiver assisting them before hospitalization could have their caregiver with them throughout the day. The study group included all patients who were admitted during the first wave of the COVID-19 pandemic (between March 1,2020, and June 30, 2020). This group was defined as the "social isolation group" since all outside visits were forbidden, and the social and familial support, usually provided throughout the rehabilitation program, was withheld. The control group comprises all patients with hip fractures who were admitted in the respective time periods during the previous 2 years, that is, March 1, 2018, to June 30, 2018, and March 1, 2019, to June 30,2019 . These patients were often visited and cared for by relatives and personal caregivers, as mentioned above. Excluded were patients who did not complete the rehabilitation program due to admission to a general hospital or a skilled nursing ward with an acute medical condition and those who died during the program or unexpectedly terminated rehabilitation.

\section{Rehabilitation Setting}

The multidisciplinary team provided medical, nursing, physical, occupational, and social work interventions. Rehabilitation 
Table 1. Pandemic group: a comparison between patients who completed rehabilitation and those who did not, regarding demographic, clinical, and functional characteristics

\begin{tabular}{lllc}
\hline & $\begin{array}{l}\text { Completed } \\
\text { rehabilitation } \\
N=36\end{array}$ & $\begin{array}{l}\text { Did not complete } \\
\text { rehabilitation } \\
N=6\end{array}$ & $p$ value \\
\hline Age, years (SD) & $83.3(8.5)$ & $88.0(5.8)$ & 0.202 \\
Albumin level on admission, g/dL (mean, SD) & $3.1(0.42)$ & $3.0(0.37)$ & 0.483 \\
Admission FIM score, mean (SD) & $54.8(13.8)$ & $56.2(17.5)$ & 0.838 \\
MMSE score, mean (SD) & $18.3(5.5)$ & $19.3(5.7)$ & 0.757 \\
\hline
\end{tabular}

SD, standard deviation; N, number; FIM, functional independence measure; MMSE, Mini-Mental State Examination.

care included the following: (1) 30-45 min of individual physiotherapy (i.e., improving transfers, walking the length of a room, climbing stairs, and equilibrium and joint range of motion), 2-3 times a week; (2) 30-40 min of physical therapy in an adapted fitness room, including muscle resistance training of major muscle groups ( 2 sets of $8-12$ repetitions), aerobic training on a treadmill with and without partial weight support, and riding a stationary bicycle, 2-3 times a week; and (3) 30-45 min of individual occupational therapy (improvement of basic activities of daily living $[\mathrm{ADL}])$, cognitive evaluation and stimulation, safety education, and learning to use assistive devices, 3 times a week. Weekly meetings were held by the multidisciplinary team to evaluate patients' progress. This framework did not change at the time of the COVID-19 pandemic.

\section{Measures}

Functional, clinical, and demographic data of patients admitted to rehabilitation were collected from their medical files. Functional variables included the functional independence measure (FIM) instrument [16] - a disability measure administered upon admission and discharge. The FIM comprises 18 parameters, each rated on a scale of 1 to 7 according to the degree of assistance required to perform a specific activity in 3 domains: ADL (8 parameters), mobility (5 parameters), and cognitive function (5 parameters). The motor FIM (mFIM) includes 13 parameters of ADL and mobility. The maximal total score for the FIM is 126 and for the mFIM, 91. The patient's FIM score was assessed during multidisciplinary team meetings by physicians and trained physical and occupational therapists. Cognitive function was measured by the Mini-Mental State Examination (MMSE) [17], administered by OTs. Depressive symptoms were assessed by the Geriatric Depression Scale (GDS) [18].

Clinical variables included comorbidities, BMI, and albumin levels on admission. Demographic variables included age, sex, residency (home vs. nursing home), presence of a live-in caregiver before admission, and education (elementary $<10$ years and higher education $\geq 10$ years). Data relating to the fracture included fracture type (extracapsular and intracapsular), fracture side (right vs. left), type of treatment (operative vs. conservative treatment), and days passed from surgery to rehabilitation (latency time). Length of stay was defined as the number of days patients resided in the rehabilitation center.

Rehabilitation under Social Isolation
Functional gain was determined as an FIM score change (discharge score minus admission score). The relative functional gain (mFIM effectiveness) achieved on the mFIM score was calculated using the Montebello Rehabilitation Factor Score (MRFS) [19, 20], designed to overcome the problem of floor and ceiling effects and to estimate each patient's specific potential for improvement. The mFIM effectiveness was calculated as the mFIM score change (discharge mFIM score minus admission mFIM score) divided by the mFIM maximum score (91) minus the mFIM admission score.

\section{Statistical Analysis}

Statistical analysis was performed using the IBM SPSS Statistics for Windows (Version 25.0; IBM Corp., Armonk, NY, USA). Data regarding the study group (reported separately for those who completed rehabilitation and those who did not) and the control group were expressed as mean \pm SD or frequencies. Favorable functional gain was defined as mFIM effectiveness greater than median score. The associations between social isolation during COVID-19 pandemic and various demographic and clinical parameters were assessed by analysis of variance and the $\chi^{2}$ test. The significance level was set at $p<0.05$. A multiple linear regression model was used to estimate the association between social isolation during COVID-19 pandemic and discharge FIM score, after controlling for sociodemographic characteristics and chronic diseases. A logistic regression analysis assessed the association between social isolation and achievement of favorable rehabilitation outcomes (mFIM effectiveness $>$ median score). Since the study was based on full electronic records of patients who completed their rehabilitation program, missing data were not an issue of concern. The authors followed the Strengthening the Reporting of Observational Studies in Epidemiology (STROBE) [21] checklist for this publication.

\section{Results}

Forty-two hip-fractured patients, aged 65 years and older, were admitted to our postacute geriatric rehabilitation ward during the social isolation period (March-June 2020). In comparison with former years, there was a decline of $>30 \%$ in the number of patients suffering from 


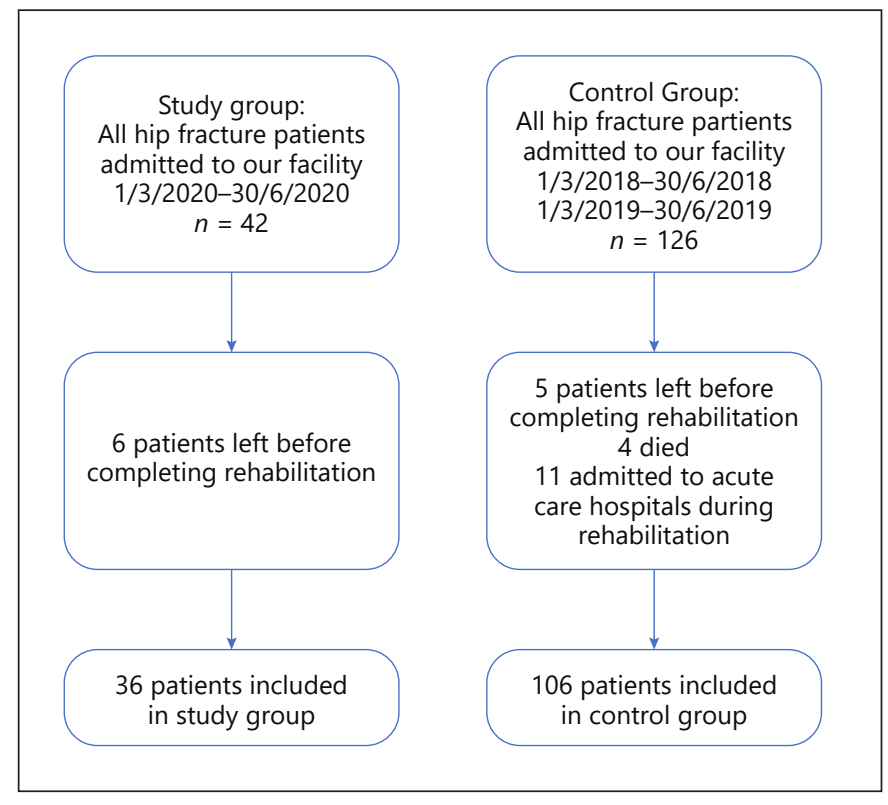

Fig. 1. Study flowchart. Rehabilitation under social isolation.

hip fractures admitted to our facility during the first wave of the pandemic, who underwent rehabilitation under social isolation from families and main caregivers. The difference in numbers between study arms is a direct result of a dramatic reduction in total occupancy rates (for all admission causes) in our facility during the first wave of the pandemic: during March-June 2020, overall occupancy was 78\%, while during parallel time frames in 2018 and 2019 , it was $99 \%$. Six patients did not complete their rehabilitation process and therefore were excluded from the study. No significant differences were found between patients who completed rehabilitation $(n=36)$ and those who did not $(n=6)$, regarding demographic, clinical, and functional characteristics (Table 1).

The control group comprises 126 patients admitted at the respective periods in 2018 and 2019 (e.g., March 1, 2018, to June 30, 2018, and March 1, 2019, to June 30, 2019), of whom 106 were included in final analysis. Five patients in the control group left the program before finishing the rehabilitation process, 4 died, and 11 were transferred to an acute care facility due to an exacerbation of their medical conditions (as shown in Fig. 1).

Table 2 presents the demographic, clinical, and functional characteristics of the 2 patient groups. Patients' demographics did not differ between groups, except for greater prevalence of higher education in the control group ( $p=0.024$ ). Comorbidities, including depression, were similar in both groups, except for higher dementia and peripheral arterial disease rates in the pandemic group ( $p=0.001$ and $p=0.046$, respectively). Regarding functional characteristics at baseline, patients in the pandemic group had significantly lower FIM scores and mFIM score upon admission compared to patients in parallel periods ( 0.016 and 0.045 , respectively). However, prefracture mFIM score did not differ between groups (Table 2).

Table 3 shows the rehabilitation outcomes of the two patient groups. All rehabilitation outcome measures were similar between groups. Multiple linear regression analysis revealed that undergoing rehabilitation under social isolation did not impact the discharge FIM score (for full analysis, see online suppl. Table 1; for all online suppl. material, see www.karger.com/doi/10.1159/000513598). We carried out a logistic regression analysis of a favorable rehabilitation achievement, defined as mFIM efficiency $>0.33$ (median score). This analysis showed that undergoing rehabilitation under social isolation was not associated with the chances of achieving a favorable rehabilitation outcome (for full analysis, see online suppl. Table 2).

\section{Discussion}

In this retrospective study, we present the rehabilitation outcomes of patients with hip fractures who were admitted to a geriatric postacute rehabilitation facility during the first wave of the COVID-19 pandemic under complete social isolation from caregivers and loved ones. During March-June 2020, the occupancy rate in our facility dropped from $99 \%$ in previous years to $78 \%$. Several explanations could be offered: during the first wave of the pandemic, patients and their families were informed in advance that once transferred to the rehabilitation facility, patients will not be able to meet with outside visitors at all, until discharged. Furthermore, in prepandemic time, it was very common for patients who had a live-in, full-time caregiver before the fracture to stay with that caregiver at our rehabilitation facility for most of the day. The caregiver offered great support, both emotionally and in ADL. During the first wave of the pandemic, outside caregivers were not allowed to enter the facility. We believe the ban on outside visitors was one of the causes for the lower occupancy rates. Another contributing factor might have been the general apprehension regarding visiting medical facilities the public felt during the pandemic, driven by fear of contracting SARS-CoV-2. Israeli health services offer eligible individuals with hip fractures the option of home rehabilitation if they have 
Table 2. Demographic, clinical, and functional characteristics of 36 hip-fractured patients who underwent rehabilitation under social isolation during March-June 2020, in comparison with patients from parallel time during 2018 and 2019

\begin{tabular}{|c|c|c|c|}
\hline & $\begin{array}{l}\text { Patients admitted } \\
\text { during social isolation } \\
(n=36)\end{array}$ & $\begin{array}{l}\text { Patients admitted } \\
\text { during previous time } \\
\text { periods }(n=106)\end{array}$ & $\begin{array}{l}\text { Statistics and } \\
p \text { value }\end{array}$ \\
\hline \multicolumn{4}{|l|}{ Demographic characteristics } \\
\hline Age, years (SD) & $83.3 \pm 8.5$ & $83.5 \pm 7.8$ & $p=0.16(-2.88$ to 3.20$)$ \\
\hline Males, $N(\%)$ & $10(27.8)$ & $21(19.8)$ & $\chi^{2}=0.99, p=0.353$ \\
\hline Higher education ( $\geq 10$ years), $N(\%)$ & $18(50.0)$ & $76(71.7)$ & $\chi^{2}=5.65, p=0.024$ \\
\hline Residency (home), N(\%) & $35(97.2)$ & $102(96.2)$ & $\chi^{2}=0.08, p=1,000$ \\
\hline \multicolumn{4}{|l|}{ Surgical characteristics } \\
\hline Intracapsular fracture, $N(\%)$ & $11(30.6)$ & $42(39.6)$ & $\chi^{2}=0.94 p=0.426$ \\
\hline Fracture side (right), $N(\%)$ & $17(47.2)$ & $58(54.7)$ & $\chi^{2}=0.61, p=0.447$ \\
\hline Treatment type (operation), $N(\%)$ & $36(100.0)$ & $101(95.3))$ & $\chi^{2}=1.76, p=0.330$ \\
\hline Time from surgery to rehabilitation, days (SD) & $8.0(5.7)$ & $7.9(5.4)$ & $\widehat{U}=-1.97 p=0.845$ \\
\hline Congestive heart failure, $N(\%)$ & $7(19.4)$ & $13(12.3)$ & $\chi^{2}=1.15, p=0.281$ \\
\hline Diabetes mellitus, $N(\%)$ & $17(47.2)$ & $38(35.8)$ & $\chi^{2}=1.46, p=0.241$ \\
\hline Hypertension, $N(\%)$ & $24(66.7)$ & $84(79.2)$ & $\chi^{2}=2.34, p=0.174$ \\
\hline Cerebrovascular accident, $N(\%)$ & $7(19.4)$ & $17(16.0)$ & $\chi^{2}=0.22, p=0.615$ \\
\hline Peripheral arterial disease, $N(\%)$ & $7(19.4)$ & $7(6.6)$ & $\chi^{2}=4.99, p=0.046$ \\
\hline Parkinson's disease, $N(\%)$ & $1(2.8)$ & $6(5.7)$ & $\chi^{2}=0.48, p=0.679$ \\
\hline Dementia, $N(\%)$ & $31(86.1)$ & $58(54.7)$ & $\chi^{2}=11.32, p=0.001$ \\
\hline Depression, $N(\%)$ & $9(25.0)$ & $33(31.1)$ & $\chi^{2}=0.48, p=0.533$ \\
\hline COPD, $N(\%)$ & $6(16.7)$ & $21(19.8)$ & $\chi^{2}=0.17, p=0.808$ \\
\hline \multicolumn{4}{|l|}{ Functional characteristics at baseline } \\
\hline Prefracture mFIM score & $72.4(13.9)$ & $71.2(11.4)$ & $\mathrm{U}=-803, p=0.422$ \\
\hline Admission FIM score & $54.8(13.8)$ & $62.0(16.8)$ & $\mathrm{U}=-2.40, p=0.016$ \\
\hline
\end{tabular}

$\mathrm{SD}$, standard deviation; N, number; BMI, body mass index; COPD, chronic obstructive pulmonary disease; FIM, functional independence measure; mFIM, motor FIM; MMSE, Mini-Mental State Examination; GDS, Geriatric Depression Scale. $p<0.05$ considered significant.

appropriate home support. Patients could have chosen to avoid social isolation and opted for home rehabilitation. A third possible explanation is that the older population were under social isolation, hence leaving their homes a lot less than usual. Fewer outings might mean fewer falls outside the home, leading to a lower incidence of hip fractures, although generally most hip fracture occurs due to falls around the house [22]. We do not yet have formal information regarding the incidence of hip fracture during the first wave of the pandemic in Israel. Worldwide reports are heterogeneous: a multicentric study from the UK demonstrated similar fracture rates with a greater tendency towards nonoperative treatment [23]. More reports from the UK [24] and Spain [25] stated the same. Other groups reported a decline in hip fractures rates $[26$, 27].

Our analysis demonstrated that patients who were admitted to our facility during the COVID-19 pandemic were more severely deconditioned and less cognitively intact than those in previous years. This is demonstrated by significantly higher rates of dementia and lower FIM scores and mFIM scores upon admission, compared with the control group. Nonetheless, prefracture mFIM scores did not differ between groups. A possible explanation is 
Table 3. Rehabilitation outcomes of 36 hip-fractured patients who underwent rehabilitation under social isolation during March-June 2020, in comparison with patients from parallel time during 2018 and 2019

\begin{tabular}{llll}
\hline & $\begin{array}{l}\text { Patients admitted during } \\
\text { social isolation }(n=36)\end{array}$ & $\begin{array}{l}\text { Patients admitted during } \\
\text { previous time periods } \\
(n=106)\end{array}$ & $\begin{array}{l}\text { Statistics and } \\
p \text { value }\end{array}$ \\
\hline Discharge FIM score, mean (SD) & $73.6(20.0)$ & $79.7(20.6)$ & $\mathrm{U}=-1.60, p=0.109$ \\
Discharge mFIM score, mean (SD) & $51.0(15.7)$ & $55.7(15.7)$ & $\mathrm{U}=-1.55, p=0.122$ \\
FIM score change, mean (SD) & $18.6(12.1)$ & $17.4(11.0)$ & $\mathrm{U}=-0.35, p=0.728$ \\
mFIM score change, mean (SD) & $18.8(12.1)$ & $16.4(10.1)$ & $\mathrm{U}=-0.23, p=0.822$ \\
mFIM effectiveness, mean (SD) & $30.3(20.4)$ & $33.5(20.8)$ & $\mathrm{U}=-0.85, p=0.396$ \\
LOS, mean (SD) & $36.5(9.1)$ & $36.5(12.9)$ & $\mathrm{U}=-0.46, p=0.647$ \\
Discharge destination (home), mean (SD) & $34(94.4)$ & $94(88.7)$ & $\chi^{2}=1.00, p=0.518$ \\
Home aid at discharge (partial), mean (SD) & $10(27.8)$ & $36(34.0)$ & $\chi^{2}=0.47, p=0.543$ \\
\hline
\end{tabular}

SD, standard deviation; FIM, functional independence measure; mFIM, motor FIM; LOS, length of stay.

that patients with severe deconditioning, as expressed by lower FIM and mFIM scores upon admission, are less likely to choose home rehabilitation. One of the reasons is that these severely deconditioned individuals might need the help of 2 caregivers or even a mechanical lift to perform transfers during postoperative rehabilitation. Since most patients do not have such facilities at home, their only option might be to be transferred to a postacute rehabilitative facility. A less deconditioned patient, requiring less help in ADL, might chose to undergo rehabilitation at home. Patients who unexpectedly terminated rehabilitation during the COVID-19 first wave were not significantly different from those who completed rehabilitation, regarding clinical and functional characteristics, thus limiting the chance of potential bias due to dropout.

Our study shows that although patients admitted during the COVID-19 pandemic were more severely deconditioned and with poorer cognitive function than the control group, no significant differences in their rehabilitation achievements were found. Thus, our null hypothesis relating to the effect of social isolation was rejected. Social distancing has been chosen by many countries as a tool of suppressing the outbreak of SARS-CoV-2 [28, 29]. Public health interventions such as restrictions of mass gatherings, school closures, and measures of social distancing have been proven effective in stumping epidemic growth [29-31]. It is well established that the 65 years and older population is at the highest risk for morbidity and mortality due to COVID-19 [4, 32, 33], and evidence is already gathering to suggest that hip-fractured patients infected with SARS-CoV-2 suffer from higher mortality rates when compared to noninfected individuals with hip fractures [34]. Geriatric facilities are vulnerable to SARS-CoV-2 out- breaks, and in many parts of the world, most COVID-19 deaths occur in long-term care facilities [35]. Under such conditions, medical institutions must take all necessary measures to make sure their patients are not exposed to SARS-CoV-2. This understanding was standing at the base of the decision to ban outside visitors from our facility.

Nevertheless, it has been long known that social isolation of the older population carries detrimental effects on both physical and mental health. Social isolation is an independent risk factor for malnutrition [36]. Loneliness increases risk of developing cardiovascular diseases, independently of traditional cardiovascular disease risk factors [37]; it is also adversely associated with different aspects of functional status [38]. Both loneliness and social isolation are associated with decreased cognitive function [39]. Finally, older individuals under social isolation are more likely to develop depression [40]. Shortly into the COVID-19 pandemic, worried voices in the medical community started raising concerns regarding the detrimental effect of social distancing on mental and physical health of the older population $[6,7,41,42]$. It is a valid question whether undergoing rehabilitation under isolation from families and main caregivers might impair outcomes. In this study, we found that these circumstances did not impact rehabilitation achievements of individuals with hip fractures. One might suggest that the same measures of social distancing that kept patients from their families and loved ones offered a feeling of protectiveness and comfort for the patients. Knowing our institute is in complete lockdown for all outside visitors gave some reassurance for older patients who watched worriedly how the COVID-19 pandemic claims victims, especially among the 65 and older population $[3,4]$. To support this 
idea even further, we draw attention to the fact that no significant differences were found between the GDS scores or length of stay of the two study groups. This suggests that isolated patients did not suffer from depression more than the control group, nor did they cut their stay short due to social isolation.

Our study has several limitations: first and foremost, the relatively small size of our cohort, stemming from the low occupancy during the first wave of the pandemic (as mentioned above). Furthermore, the study population comprises patients hospitalized in only one ward, in one postacute geriatric rehabilitation center, a fact that may weaken the generalizability of our results. As a retrospective study, it has inherent limitations such as available outcome measures from patients' files and missing data that might have introduced bias to the results. Due to the study group's small size, some differences between groups might not have been recognized. Nevertheless, since we used meticulous data collection, our database is of high quality, a fact which offsets some of the biases mentioned. Due to the unusual nature of the COVID-19 pandemic, which has an ongoing worldwide effect on healthcare worldwide, we believe this report offers important information for both policy makers and geriatric rehabilitation experts.

\section{Conclusion}

We presented data from a retrospective cohort study of individuals with hip fractures undergoing rehabilitation under social isolation during the COVID-19 pan- demic. To the best of our knowledge, social isolation did not have a detrimental effect on the rehabilitation outcomes of these patients. More evidence on the matter must be gathered and presented.

\section{Statement of Ethics}

The Rabin Medical Center Institutional Review Board approved the study (0468-20-RMC). Since the study was based on full electronic records of patients who completed their rehabilitation program, missing data were not an issue of concern. Due to the prospective design of this study, informed consent was deemed unnecessary by the Institutional Ethics Committee.

\section{Conflict of Interest Statement}

The authors have no conflicts of interest to declare.

\section{Funding Sources}

This publication received no funding.

\section{Author Contributions}

Y.L.W. conceptualized and designed the study, interpreted data, and wrote the manuscript; Y.L. and M.M. reviewed the manuscript; A.H. conceptualized and designed the study, curated and analyzed the data, interpreted it, and reviewed the manuscript. All authors gave their approval to the final version. This work was presented as a poster at the Israeli Geriatric Society conference, September 2020.

\section{References}

1 Chan JF, Yuan S, Kok KH, To KK, Chu H, Yang J, et al. A familial cluster of pneumonia associated with the 2019 novel coronavirus indicating person-to-person transmission: a study of a family cluster [Internet]. Lancet. $2020 \mathrm{Feb} ; 395(10223): 514-23$.

2 Wang D, Hu B, Hu C, Zhu F, Liu X, Zhang J, et al. Clinical characteristics of 138 hospitalized patients with 2019 novel coronavirus-infected pneumonia in Wuhan, China [Internet]. JAMA. 2020 Feb;323(11):1061-69.

$3 \mathrm{Wu}$ Z, McGoogan JM. Characteristics of and important lessons from the coronavirus disease 2019 (COVID-19) outbreak in China: summary of a report of 72314 cases from the Chinese Center for Disease Control and Prevention. JAMA. 2020 Apr;323(13):1239-42.
4 Zhou F, Yu T, Du R, Fan G, Liu Y, Liu Z, et al. Clinical course and risk factors for mortality of adult inpatients with COVID-19 in Wuhan, China: a retrospective cohort study. Lancet. 2020 Mar;395(10229):1054-62.

5 Nevo.co.il [Internet]. [cited 2020 Jul 17]. Available from: https://www.nevo.co.il/law_ $\mathrm{html} / \mathrm{law} 01 / 502$ 230.htm.

6 Armitage R, Nellums LB. COVID-19 and the consequences of isolating the elderly. Lancet Public Health. 2020 May;5(5):e256.

7 Plagg B, Engl A, Piccoliori G, Eisendle K. Prolonged social isolation of the elderly during COVID-19: between benefit and damage. Arch Gerontol Geriatr. 2020 Jul-Aug; 89: 104086.

8 Ni Z, Lebowitz ER, Zou Z, Wang H, Liu H, Shrestha R, et al. Response to the COVID-19 outbreak in urban settings in China. J Urban Health. 2021 Feb;98(1):41-52.
9 McCabe M. Impact of family presence in the healthcare setting. 2014. Senior Honors Theses. 459. Available from: https://digitalcommons.liberty.edu/honors/459.

10 Haines KJ. Engaging families in rehabilitation of people who are critically ill: an underutilized resource. Phys Ther. 2018 Sep;98(9): 737-44.

11 Foster AM, Armstrong J, Buckley A, Sherry J, Young T, Foliaki S, et al. Encouraging family engagement in the rehabilitation process: a rehabilitation provider's development of support strategies for family members of people with traumatic brain injury. Disabil Rehabil. 2012 Nov;34(22):1855-62.

12 Creasy KR, Lutz BJ, Young ME, Stacciarini JM. Clinical implications of family-centered care in stroke rehabilitation. Rehabil Nurs. 2015;40(6):349-59. 
13 De Biase S, Cook L, Skelton DA, Witham M, Ten Hove R. The COVID-19 rehabilitation pandemic. Age Ageing. 2020 Aug 24;49(5): 696-700.

14 Simpson R, Robinson L, Rehabilitation after critical illness in people with COVID-19 infection [Internet]. Am J Phys Med Rehabil. 2020 Jun;99(6):470-4.

15 Grabowski DC, Joynt Maddox KE. Postacute care preparedness for COVID-19: thinking ahead. JAMA. 2020 May;323(20):2007-8.

16 Granger CV, Hamilton BB. The uniform data system for medical rehabilitation report of first admissions for 1992. Am J Phys Med Rehabil. 1994 Feb;73(1):51-5.

17 Folstein MF, Folstein SE, McHugh PR. "Minimental state". A practical method for grading the cognitive state of patients for the clinician. J Psychiatr Res. 1975 Nov;12(3):189-98.

18 Yesavage JA, Brink TL, Rose TL, Lum O, Huang V, Adey M, et al. Development and validation of a geriatric depression screening scale: a preliminary report. J Psychiatr Res. 1982-1983;17(1):37-49.

19 Heruti RJ, Lusky A, Barell V, Ohry A, Adunsky A. Cognitive status at admission: Does it affect the rehabilitation outcome of elderly patients with hip fracture? Arch Phys Med Rehabil. 1999 Apr;80(4):432-6.

20 Rolland Y, Pillard F, Lauwers-Cances V, Busquère F, Vellas B, Lafont C. Rehabilitation outcome of elderly patients with hip fracture and cognitive impairment. Disabil Rehabil. 2004 Apr;26(7):425-31.

21 von Elm E, Altman DG, Egger M, Pocock SJ, Gøtzsche PC, Vandenbroucke JP. The Strengthening the Reporting of Observational Studies in Epidemiology (STROBE) statement: guidelines for reporting observational studies. Ann Intern Med. 2007 Oct;147(8): 573-7.

22 Aharonoff GB, Dennis MG, Elshinawy A, Zuckerman JD, Koval KJ. Circumstances of falls causing hip fractures in the elderly. J Orthop Trauma. 2003 Sep;17(8 Suppl l):S22.

23 Hall AJ, Clement ND, Farrow L, MacLullich AMJ, Dall GF, Scott CEH, et al. IMPACT-
Scot report on COVID-19 and hip fractures: a multicentre study assessing mortality, predictors of early SARS-CoV-2 infection, and the effects of social lockdown on epidemiology. Bone Joint J. 2020 Sep;102-B(9):1219-28.

24 Park C, Sugand K, Nathwani D, Bhattacharya R, Sarraf KM. Impact of the COVID-19 pandemic on orthopedic trauma workload in a London level 1 trauma center: the "golden month": the COVid Emergency Related Trauma and orthopaedics (COVERT) Collaborative. Acta Orthop. 2020;91(5):556-61.

25 Nuñez JH, Sallent A, Lakhani K, Guerra-Farfan E, Vidal N, Ekhtiari S, et al. Impact of the COVID-19 pandemic on an emergency traumatology service: experience at a tertiary trauma centre in Spain. Injury. 2020 Jul;51(7): 1414-8.

26 Wong KC, Cheok JWG, Tay KXK, Koh SB, Howe TS. Where have all the hip fractures gone? Osteoporos Int. 2020 Oct;31(10):2057-8.

27 Minarro JC, Zamorano-Moyano C, UrbanoLuque MT, Arenas-de Larriva AP, IzquierdoFernández A, Quevedo-Reinoso R. Is COVID-19 affecting the incidence of hip fractures? Injury. 2020;51(10):2329.

28 Lewnard JA, Lo NC. Scientific and ethical basis for social-distancing interventions against COVID-19. Lancet Infect Dis. 2020 Jun;20(6): 631-3.

29 Pan A, Liu L, Wang C, Guo H, Hao X, Wang $\mathrm{Q}$, et al. Association of public health interventions with the epidemiology of the COVID-19 outbreak in Wuhan, China. JAMA. 2020 May 19;323(19):1915-23.

30 Jüni P, Rothenbühler M, Bobos P, Thorpe KE, da Costa BR, Fisman DN, et al. Impact of climate and public health interventions on the COVID-19 pandemic: a prospective cohort study. CMAJ. 2020 25;192(21):E566-73.

31 Flaxman S, Mishra S, Gandy A, Unwin HJT, Mellan TA, Coupland $\mathrm{H}$, et al. Estimating the effects of non-pharmaceutical interventions on COVID-19 in Europe. Nature. 2020 Aug; 584(7820):257-61

32 CDC COVID-19 Response Team. Severe outcomes among patients with coronavirus dis- ease 2019 (COVID-19) - United States, February 12-March 16, 2020. MMWR Morb Mortal Wkly Rep. 2020 Mar;69(12):343-6.

33 International Severe Acute Respiratory and Emerging Infections Consortium (ISARIC). 29.

34 Muñoz Vives JM, Jornet-Gibert M, CámaraCabrera J, Esteban PL, Brunet L, DelgadoFlores L, et al. Mortality rates of patients with proximal femoral fracture in a worldwide pandemic: preliminary results of the Spanish HIP-COVID Observational Study. J Bone Joint Surg Am. 2020 Jul;102(13):e69.

35 Lau-Ng R, Caruso LB, Perls TT. COVID-19 deaths in long term care facilities: a critical piece of the pandemic puzzle. J Am Geriatr Soc. 2020 Sep;68(9):1895-8.

36 Boulos C, Salameh P, Barberger-Gateau P. Social isolation and risk for malnutrition among older people. Geriatr Gerontol Int. 2017 Feb; 17(2):286-94.

37 Valtorta NK, Kanaan M, Gilbody S, Hanratty B. Loneliness, social isolation and risk of cardiovascular disease in the English Longitudinal Study of Ageing. Eur J Prev Cardiol. 2018; 25(13):1387-96.

38 Shankar A, McMunn A, Demakakos P, Hamer M, Steptoe A. Social isolation and loneliness: prospective associations with functional status in older adults. Health Psychol. 2017 Feb;36(2):179-87.

39 Lara E, Caballero FF, Rico-Uribe LA, Olaya B, Haro JM, Ayuso-Mateos JL, et al. Are loneliness and social isolation associated with $\operatorname{cog}$ nitive decline? Int J Geriatr Psychiatry. 2019; 34(11):1613-22.

40 Förster F, Stein J, Löbner M, Pabst A, Angermeyer MC, König H-H, et al. Loss experiences in old age and their impact on the social network and depression: results of the Leipzig Longitudinal Study of the Aged (LEILA 75+). J Affect Disord. 2018 Dec;241:94-102.

41 Jawaid A. Protecting older adults during social distancing. Science. 2020 Apr;368(6487): 145.

42 Brooke J, Jackson D. Older people and COVID-19: isolation, risk and ageism. J Clin Nurs. 2020;29(13-14):2044-6. 